\title{
La función de la Oficina Nacional para el Control del Tabaco en México
}

\author{
Justino Regalado-Pineda, M en C, ${ }^{(1,2)}$ Carlos José Rodríguez-Ajenjo, MD. ${ }^{(3)}$
}

\author{
Regalado-Pineda J, Rodríguez-Ajenjo CJ. \\ La función de la Oficina Nacional \\ para el Contro del Tabaco en México. \\ Salud Publica Mex 2008;50 supl 3:S355-S365.
}

\section{Resumen}

El tabaquismo, como la primera causa de muerte prevenible en el mundo, impone la implementación de políticas públicas acordes a la dimensión el problema. El Convenio Marco para el Control del Tabaco (CMCT) de la Organización Mundial de la Salud establece una guía para este propósito. El solo hecho de haber firmado y ratificado el convenio no garantiza la consecución de los objetivos centrales, su éxito depende del compromiso y de las acciones implementadas por cada uno de los países que lo han adoptado. Es necesaria la construcción de las capacidades necesarias para el desarrollo de políticas sustentables de control del tabaco. La "capacidad nacional" consiste en el desarrollo de recursos humanos expertos y la creación de una estructura de ingeniería organizacional que le dé sustento a los programas sobre los que operará la implementación del tratado. La creación de la Oficina Nacional para el Control del Tabaco (ONCT) permitirá fortalecer las acciones del Gobierno Federal encaminadas a la aplicación de políticas públicas de acuerdo con los lineamientos del CMCT de manera sustentable y permanente. En este ensayo se plantea la importancia de la ONCT para la implementación de políticas públicas para el control del tabaco, las funciones que desempeñará y los objetivos a mediano y largo plazo.

Palabras claves: tabaco; políticas públicas; contaminación por humo de tabaco; cese del tabaquismo; México
Regalado-Pineda J, Rodríguez-Ajenjo CJ.

The role of the National Office

for Tobacco Control in Mexico.

Salud Publica Mex 2008;50 suppl 3:S355-S365.

\begin{abstract}
Tobacco smoking as the first cause of preventable dead in the world requires the implementation of proper public policies. The WHO Framework Convention on Tobacco Control (FCTC) sets the basis for this purpose at the national level. But the successful implementation of FCTC depends on a series of actions of every single member of the convention for the development of National Capacity. Building capacity implies the construction of human resources and organizational engineering or "institutional building" in order to enforce and guarantee the Plan of Action for the implementation of FCTC. The creation of the National Office for Tobacco Control of México will allow the enforcement of Mexican government initiatives on implementation of policies for tobacco control, according with FCTC on a sustainable and permanent platform. This essay presents the importance on the office, their main functions and their medium and long term objectives
\end{abstract}

Key words: tobacco; public policies; tobacco smoke pollution; smoking cessation; Mexico

(I) Departamento de Investigación en Tabaquismo, Instituto Nacional de Enfermedades Respiratorias, México.

(2) Oficina Nacional para el Control del Tabaco. Consejo Nacional Contra las Adicciones. Secretaría de Salud, México.

(3) Secretario Técnico. Consejo Nacional Contra las Adicciones. Secretaría de Salud, México. 
E tabaco constituye en la actualidad la principal causa de muerte prevenible en el mundo, con una tasa de letalidad mayor a los 5 millones de muertes al año, lo que equivale a cerca de 14000 muertes diarias, ${ }^{1,2}$ una tasa mayor a la mortalidad asociada a enfermedades como la tuberculosis, infección por VIH/SIDA o malaria juntas. ${ }^{1}$ En México, ocurren aproximadamente 65000 muertes al año entre personas fumadoras por enfermedades asociadas con el tabaquismo. ${ }^{3}$

En la actualidad, el tabaco es el único producto de consumo de venta legal que daña a todos los individuos que se exponen de manera voluntaria o involuntaria a su humo y que además, mata prematuramente a quienes lo utilizan de acuerdo con las instrucciones del fabricante, en promedio 15 años antes. ${ }^{4,5}$ Por si fuera poco, los fumadores no son los únicos que ven afectada su salud como consecuencia de la inhalación del humo de sus cigarrillos, todas las personas que se exponen al humo de tabaco de segunda mano (tabaquismo pasivo o involuntario) corren el riesgo de enfermar y morir prematuramente como consecuencia de las mismas enfermedades que afectan a los fumadores. ${ }^{6,7}$

La magnitud de la epidemia del tabaquismo constituye un fenómeno creciente donde la tendencia es al aumento en el número de fumadores en todo el mundo. ${ }^{8}$ Este aumento se verá más pronunciado en los países en desarrollo, dado que la mayoría de ellos se encuentran en fases más tempranas de la epidemia del tabaco. ${ }^{9}$ Por otro lado, el consumo de tabaco en los países en desarrollo crece de manera más acelerada en parte debido al crecimiento estable de su población y debido a las acciones implementadas por la industria tabacalera. ${ }^{9}$ En el caso de México, la tendencia en el consumo de tabaco en la población mexicana se ha mantenido estable durante los últimos 15 años (cuadro I, figura 1), observándose, en general, una tendencia hacia el abandono del consumo en la población general, actualmente la prevalencia de consumo de tabaco en la población entre 12 y 65 años es de 26.4 por ciento. ${ }^{10}$ Sin embargo, el número total de fumadores en el país se ha incrementado desde aproximadamente 9 millones de fumadores en 1988, a cerca de 16 millones de fumadores en 2002 debido al crecimiento de la población general del país. ${ }^{10,11}$ La población masculina se ha mantenido estable con una prevalencia de aproximadamente 39\% desde la primer Encuesta Nacional de Adicciones (ENA). La población de mujeres fumadoras ha crecido discretamente desde $14.4 \%$ en 1988 , a $16.1 \%$ en la última ENA 2002, dato consistente con la tendencia mundial hacia el incremento de la prevalencia de tabaquismo en mujeres, especialmente adolescentes ${ }^{9,12-14}$ e imponiendo un matiz diferente al carácter de la epidemia en los años por venir donde, de continuar esta tendencia, observa- remos la emergencia de un mayor número de casos de enfermedades relacionadas con el consumo de tabaco en mujeres.

El impacto económico del tabaquismo es excesivo considerando los altos costos en que incurren los pacientes cuando se ven afectados por alguna de las múltiples enfermedades asociadas con el tabaquismo, dejando tras de sí una gran carga de sufrimiento, enfermedad, muerte y cuantiosas pérdidas económicas derivadas de la atención médica y pérdida de productividad y competitividad. Lo anterior deriva en un empobrecimiento de la sociedad en su conjunto, efecto que se ve más pronunciado en los países en desarrollo, especialmente en los estratos más desfavorecidos. ${ }^{15-22}$

\begin{tabular}{|c|c|c|c|}
\hline & Cuadro I & & \\
\hline Tendenc & $\begin{array}{l}\text { N LA PREVALenCIA De } \\
\text { en MÉXICO }\end{array}$ & ABAQUIS & \\
\hline $\begin{array}{c}\text { Encuesta Nacional } \\
\text { de Adicciones }\end{array}$ & $\begin{array}{c}\text { Prevalencia } \\
\text { Fumadores (área urbana) }\end{array}$ & Hombres & Mujeres \\
\hline 1988 & $25.8 \%$ & $38.3 \%$ & $14.5 \%$ \\
\hline 1993 & $25.1 \%$ & $38.3 \%$ & $14.2 \%$ \\
\hline 1998 & $27.7 \%$ & $42.9 \%$ & $16.3 \%$ \\
\hline 2002 & $26.4 \%$ & $39.1 \%$ & $16.1 \%$ \\
\hline
\end{tabular}

Fuente: Encuesta Nacional de Adicciones/SSA, 1988, 1993, 1998 y 2002

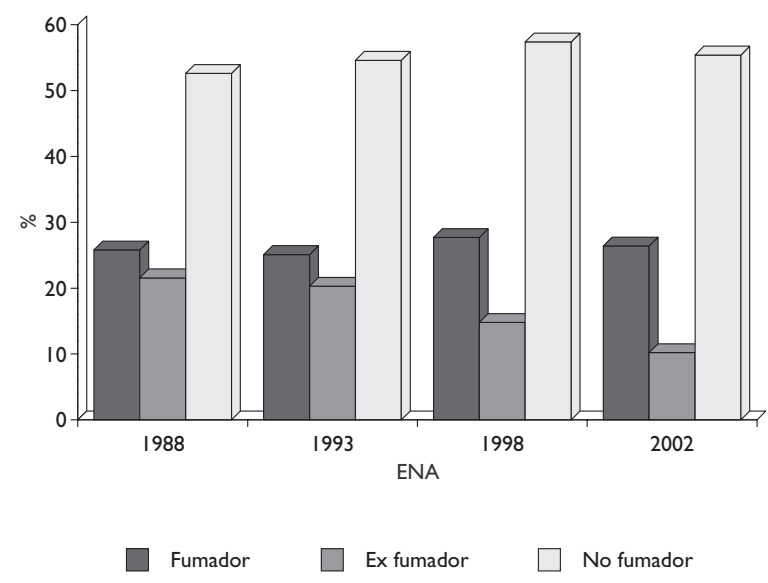

Figura I. Prevalencia de consumo de tabaco en MéxiCO, 1988, 1993, 1998, 2002. Encuesta NACIONAL dE Adicciones. MéXICo 
El conocimiento sobre los efectos negativos para la salud ocasionados por el consumo crónico de productos del tabaco se encuentra ampliamente difundido por diferentes medios, tanto científicos como de comunicación masiva. ${ }^{5,7}$

Las dimensiones de la epidemia del tabaco en el mundo demandan soluciones a fondo. El establecimiento de un tratado internacional propuesto por la Organización Mundial de la Salud (OMS) nos permite contar con una plataforma internacional para hacer frente al problema en el ámbito nacional. Tal es la infraestructura que propone el Convenio Marco para el Control del Tabaco (CMCT). ${ }^{23}$ El CMCT contiene una serie de directrices acordes con la evidencia científica más sólida para coordinar acciones globales que permitan detener una de las amenazas para la salud más significativas que se enfrenta en la actualidad..$^{24}$ Sin embargo, el solo hecho de haber firmado y ratificado semejante tratado internacional, no garantiza la consecución de los objetivos centrales, su éxito depende en gran medida del compromiso y de las acciones implementadas por cada uno de los estados que lo han adoptado. ${ }^{25}$ Desde el punto de vista de la colaboración internacional, la implementación del CMCT en cada uno de los países miembros, supone un reto en cuanto a la construcción de las capacidades necesarias para el desarrollo de políticas sustentables de control del tabaco. 26,27

En México, el CMCT fue firmado en mayo de 2003 y ratificado un año después, siendo el primer país en la región de América en ratificarlo. ${ }^{28,29}$ Entró en vigor para los primeros 40 países que depositaron el tratado firmado y ratificado por sus respectivos gobiernos ante la Asamblea General de Salud de la Organización de Naciones Unidas el 27 de febrero de 2005. A partir de su entrada en vigor, las partes se comprometen a la implementación del tratado y a la puesta en vigor de diversas acciones que permitan reducir significativamente la carga sobre el sistema de salud como consecuencia del tabaquismo.

Lo anterior implica además la construcción y el fortalecimiento de la capacidad nacional para hacer frente a tan complejo problema. Pero, ¿qué es exactamente lo que se quiere decir cuando se habla de construir la "capacidad nacional"? Consiste en el desarrollo de recursos humanos expertos en el tema y en la creación de una estructura de ingeniería organizacional que le den sustento a los programas sobre los que operará la implementación del tratado. ${ }^{30} \mathrm{El}$ aspecto institucional resulta de trascendencia tomando en cuenta, en el caso de México, que se trata de un plan de acción a largo plazo que establece el Estado Mexicano y que va más allá de la conformación de infraestructura humana y física que permita garantizar la sustentabilidad de la lucha contra el tabaco. El proceso de creación de la capacidad nacional para el combate al tabaquismo también deberá incluir en su esfera de influencia a instituciones fuera del sector público, especialmente las organizaciones de la sociedad civil. Para poder alcanzar los objetivos planteados, debemos desarrollar la habilidad de desempeñar tareas, solucionar problemas y cumplir objetivos en tres ámbitos: individual, institucional y social. ${ }^{25,31}$

A pesar de contar con información pertinente respecto de la dimensión y consecuencias de la epidemia del tabaco, todavía es frecuente encontrar cierto grado de resistencia entre la población general, incluso entre los segmentos supuestamente mejor informados de dicha población hacia medidas de control en materia de aplicación de políticas públicas para el control del tabaquismo y las enfermedades asociadas. Más aún, en la actualidad son pocos los países que cuentan con la infraestructura y recursos adecuados para implementar un programa básico para el control del tabaco. Por citar un ejemplo, vale la pena mencionar que en la región de Europa central de la OMS (WHO/EMRO por sus siglas en inglés), de los 51 países miembros, sólo 27 cuentan con un plan de acción contra el tabaco y 32 países cuentan con una estructura administrativa dedicada al control del tabaco, $45 \%$ de los países que proporcionaron datos no cuentan con un programa específico para el control del tabaco y $25 \%$ carecen de un comité multisectorial para los mismos fines; finalmente, $55 \%$ de los programas existentes se encuentran desarticulados de otros programas de salud. ${ }^{32,33}$ En la región de las Américas, de los 36 países miembros, sólo seis cuentan con una estructura dedicada al control del tabaco; 23 países cuentan con un programa de acción para el control del tabaco y 17 países cuentan con un comité multisectorial. ${ }^{33,34}$ Podemos citar casos que en los últimos años se han destacado por sus avances en el control del tabaco, como ha sucedido con Uruguay, el primer país del continente americano en alcanzar el estatus de país 100\% libre de humo de cigarro; Canadá, que cuenta con varias provincias $100 \%$ libres de humo de tabaco; Estados Unidos, que ha avanzado con varios estados libres de humo; Brasil, que en los últimos años se ha constituido en un líder indiscutible en materia de implementación de políticas públicas para el control del tabaco, y merece una mención especial el hecho de ser junto con Canadá pionero en la inclusión de pictogramas de advertencia en las cajetillas de cigarrillos. ${ }^{34}$ Otros países han emprendido esfuerzos específicos para construir la capacidad nacional requerida para hacer frente al problema, tal es el caso de Belice, Costa Rica, El Salvador, Guatemala, Honduras, Nicaragua, Panamá y Perú. ${ }^{35}$ En términos de los esfuerzos concretos realizados por países de la región se destaca el aumento neto del 
precio de los cigarrillos en países como Estados Unidos, Paraguay, México, Chile, Ecuador y Panamá. ${ }^{36}$

México ha incorporado, desde hace varios años, diversos elementos para el control del tabaco, aunque, como ha ocurrido en otros países, lo ha hecho de manera desarticulada con otros programas de salud pública. El control del tabaco y las enfermedades asociadas ha ocupado a las autoridades federales y estatales de tiempo atrás, inclusive antes de la firma y ratificación del CMCT. ${ }^{37}$ Se cuenta con diversos ordenamientos para el control del tabaco y protección de los no fumadores, tanto en el ámbito federal como en diversas leyes y reglamentos de jurisdicción estatal. Sin embargo, hace falta articular un plan de acción coordinado que permita avanzar de manera más decidida en el tema del control del tabaco.

\section{La Oficina Nacional para el Control del Tabaco}

El Consejo Nacional para las Adicciones (CONADIC) de la Secretaría de Salud federal, es la instancia encargada de la aplicación de las políticas públicas relacionadas con el uso y abuso de substancias entre las que se incluye al tabaco, alcohol y substancias ilícitas. En el momento actual no existe un área dedicada específicamente al control del tabaco. La creación de la Oficina Nacional para el Control del Tabaco (ONCT) permitirá fortalecer todas las actividades relacionadas con el control del tabaco en el territorio nacional. Tomando en cuenta las recomendaciones internacionales (cuadro II), se plantean los objetivos y funciones de la ONCT. A partir de su conformación, la tarea central de la oficina será constituirse en la instancia líder del Gobierno Federal, capaz de conducir e impulsar en todo el país la prevención y atención del tabaquismo, así como la promoción del control del tabaco. La ONCT se encargará de fortalecer las acciones del Gobierno Federal encaminadas a la aplicación de políticas públicas de acuerdo con los lineamientos del CMCT de la OMS de manera sustentable y permanente.

La ONCT se encargará de reforzar las actividades para la implementación de políticas públicas con base en el CMCT, poniendo énfasis especial en los espacios públicos $100 \%$ libres de humo de tabaco; la reglamentación del empaquetado y etiquetado de los productos del tabaco; la prohibición de la publicidad, promoción y patrocinio, implementación de estrategias de comunicación de los riesgos por consumo crónico de tabaco, así como la promoción de políticas fiscales encaminadas a reducir el consumo del tabaco en la población mexicana. La ONCT se encargará de coordinar acciones ante las distintas instancias con jurisdicción para implementar cada una de las acciones mencionadas, así como entre los diferentes integrantes del gobierno mexicano en sus tres niveles: federal, estatal y municipal.

Objetivos de la ONCT:

- Implementar el CMCT de la OMS y dar cumplimiento a las directrices acordadas por las Conferencia de las partes en tiempo y forma.

\section{Cuadro II \\ Estrategias para el desarrollo de la “cApacidad nacional” \\ Importancia}

Tarea

Definición de objetivos

\begin{tabular}{l} 
Definición de objetivos \\
\hline Desarrollo de estrategias \\
\hline Diseño de un plan de acción \\
\hline Desarrollo e implementación de políticas apropiadas \\
\hline Desarrollo de una plataforma legal y regulatoria \\
\hline Construcción de alianzas
\end{tabular}

Contacto con organizaciones de la sociedad civil

Movilización y manejo de recursos

Implementación de planes de acción

Monitoreo del avance del programa
Implica la comprensión del contexto local y nacional, así como de la habilidad para identificar grupos vulnerables

Identificar el escenario político más propicio

Basado en estrategias concertadas, resultados esperados y alcances concretos

Formulación de políticas y estrategias, monitoreo de su implementación

Adaptación de la legislación local para asegurar la implementación del CMCT de la OMS

Estrategias de consulta y comunicación con actores clave que garanticen la implementación del plan de acción

Comunicación efectiva y abogacía con actores clave dentro del sector público y privado

Garantizar adecuados recursos humanos, financieros y de otra índole necesarios para la implementación de programas costo-efectivos

Selección y capacitación adecuada de los individuos e instituciones para poner en marcha el plan de acción

Aseguramiento de los recursos necesarios para medir objetivamente los avances logrados

Fuente: Modificado de: World Health Organization. Building blocks for tobacco control: A handbook [monografía en internet]. Ginebra, Suiza, 2004 [consultado 30 abril 2008]. Disponible en http://www.who.int/tobacco/resources/publications/tobaccocontrol_handbook/en/ 
- Desarrollar Estrategia y Plan de Acción para coadyuvar en el establecimiento de la Ley General para el Control del Tabaco (LGCT) y leyes estatales para el control del tabaco.

- Definir líneas de acción en los niveles: federal, estatal y municipal.

- Implantar y dar vigencia a la LGCT en todo el país.

- Reconocimiento de la capacidad instalada estructura institucional, recursos disponibles y ámbitos preferenciales de acción. Impulsar el fortalecimiento de la capacidad nacional para el control del tabaco.

- Participación en la elaboración de la reglamentación de los productos de tabaco.

- Seguimiento de las acciones implementadas en relación con el etiquetado, promoción, venta, propaganda, publicidad, impuestos y espacios $100 \%$ libres de humo.

- Fortalecimiento de la red de servicios de cesación.

- Apoyo a la investigación en materia de comportamiento de la epidemia del tabaquismo en México, prevalencia de la adicción a la nicotina, mejores prácticas para el tratamiento de la adicción a la nicotina, entre otras.

- Vigilancia del comportamiento de la industria.

Las funciones básicas de la ONCT serán las siguientes:

- Desarrollar y proponer al CONADIC las políticas públicas para el control del tabaco, con base en la evidencia científica, la experiencia internacional y de conformidad con el CMCT.

- Fortalecer la coordinación de acciones en los órdenes de gobierno, así como promover alianzas y la participación de los sectores público, privado y social.

- Integrar y coordinar el Sistema Nacional de Información y Vigilancia para el Control del Tabaco.

- Procurar la asignación suficiente y permanente de recursos para garantizar la sustentabilidad del Programa Nacional.

- Gestionar el conocimiento técnico-científico sobre el tema.

- Consolidar y mantener la cooperación internacional dando respuesta a los requerimientos y compromisos con organismos multilaterales y regionales.

\section{Implementación de la Ley General para el Control del Tabaco}

El 26 de febrero de 2004, la Cámara de Senadores aprobó la LGCT. ${ }^{38}$ En esta ley se incorporan diversas medidas contempladas en el CMCT. Los objetivos primordiales de la ley en términos de salud pública son: reducir la prevalencia del consumo de tabaco en la población mexicana, así como reducir la exposición al humo de tabaco.

Para la adecuada implementación de la LGCT será indispensable considerar los siguientes elementos:

- Implementación en todo el territorio nacional.

- Análisis de componentes de aplicación federal y ámbitos locales.

- Implementación de estrategias de difusión y campañas sensibilización.

- Elaboración de la reglamentación. Adecuación de reglamentos, normas, bandos estatales y municipales:
o Ley estatal
o Reglamentos estatales
o Bandos municipales

- Necesidad de seguimiento y aplicación de sanciones.

- Definición de los mecanismos que permitan que la aplicación de los recursos derivados de las sanciones administrativas se inviertan en el seguimiento de la LGCT.

- Vigilancia y cumplimiento de la normatividad.

- Promoción y difusión de la regulación.

- Mecanismos de denuncia y seguimiento de denuncias.

- Integración y coordinación del Sistema Nacional de Vigilancia para el Control del Tabaco.

- Elaboración de políticas preventivas y de rehabilitación para los pacientes.

Desde su creación, la ONCT se encargará de dar seguimiento al proceso de entrada en vigor de la LGCT lo que incluye la redacción y adecuación de los reglamentos correspondientes, coordinación con las entidades federativas para la implementación de reglamentos de aplicación de la LGCT, seguimiento del proceso de entrada en vigor y cumplimiento de la LGCT. Para tales fines se establecerá colaboración estrecha con los centros estatales para el Control de las Adicciones e instancias afines, así como asociaciones médicas, cámaras empresariales, sindicatos, instituciones de educación superior, etcétera, de tal manera que se garantice la vinculación con los sectores público, privado y social que permita una adecuada comunicación y el desarrollo y fortalecimiento de capacidades de acción.

\section{Cumplimiento y ratificación del CMCT}

Derivado de la firma y ratificación del CMCT de la OMS el Estado Mexicano deberá dar cumplimiento a las disposiciones de la Conferencia de las Partes para la imple- 
mentación de medidas de empacado y etiquetado de los productos del tabaco de acuerdo con los Artículos 11 y 15 del CMCT. En el Artículo 11 se señala la necesidad de incorporar leyendas precautorias que cubran de 30 a 50\% de ambas caras de las cajetillas de cigarros, así como la inclusión de pictogramas que ilustren los daños a la salud de manera contundente. La incorporación de advertencias que señalen con claridad los peligros para la salud resulta de gran importancia para cambiar la imagen del tabaco, principalmente entre los jóvenes. Es muy importante que las personas asocien al tabaco con su capacidad de inducir adicción y las graves consecuencias para la salud que resultan de su consumo. ${ }^{9} \mathrm{La}$ ONCT se encargará de desarrollar las estrategias para dar cumplimiento a esta disposición.

De la misma manera, vigilará que las advertencias sanitarias no incluyan términos ambiguos o confusos como las palabras "suaves", "light", "bajo en alquitrán", etc. Los fabricantes de cigarrillos tradicionalmente han empleado el diseño de sus cajetillas como una plataforma para influir en sus consumidores, especialmente los más jóvenes creando vínculos de lealtad hacia la marca. La inclusión de leyendas de advertencia reduce este efecto del mercadeo. La industria tabacalera también utiliza el empaque de su producto para engañar a los consumidores mediante el empleo de términos falsos o confusos tales como "suaves", "light" o "bajo en alquitrán", ninguno de los cuales significa que el consumidor esté en menor riesgo. ${ }^{39}$ La adición de leyendas precautorias o advertencias de salud en las cajetillas tiene efectos positivos, aumenta el nivel de información de los fumadores. ${ }^{40} \mathrm{El}$ empleo de imágenes o pictogramas que ilustran de manera gráfica las consecuencias para la salud, posee aun mayor impacto y mejora la penetración de los mensajes entre población analfabeta. La ONCT propondrá el diseño a utilizar en el empaquetado y etiquetado de los productos del tabaco.

La publicidad de los productos del tabaco resulta ser uno de los medios más efectivos de la industria tabacalera para ganar nuevos adeptos, especialmente entre los más jóvenes. La industria alega que la publicidad y promoción no están dirigidas hacia la expansión del mercado o para la atracción de nuevos clientes, tan sólo pretende la redistribución del mercado entre los consumidores existentes, lo cual resulta ser falso. ${ }^{41}$ La publicidad y la promoción del tabaco aumentan las ventas mediante la inducción de los fumadores a fumar más y disminuyendo su motivación para dejar de fumar. ${ }^{42}$ Recomendaciones internacionales sugieren a los gobiernos de los países miembros, idealmente en asociación con los organismos de la sociedad civil, implementar campañas de contrapublicidad a través de todos los medios disponibles para difundir de manera extensa los daños relacionados con el consumo de tabaco. Estas campañas coadyuvan a eliminar el glamour que rodea al tabaco y expone su carácter dañino y negativo. En diferentes partes del mundo se han implementado campañas exitosas que han mostrado su utilidad para convencer a los fumadores para que abandonen el tabaco. ${ }^{43}$

Tomando en cuenta este fenómeno, el CMCT exige que las partes implementen una prohibición integral de la publicidad, la promoción y el patrocinio del tabaco. El Artículo 13 del CMCT se refiere a la publicidad, la promoción y el patrocinio del tabaco. Según esta sección, el Estado Mexicano deberá implementar medidas orientadas a los siguientes aspectos:

- Prohibir la publicidad, la promoción y el patrocinio del tabaco antes de los cinco años posteriores a la entrada en vigencia del CMCT (27 de febrero de 2010).

- Incluir la publicidad, la promoción y el patrocinio "transfronterizos" que se origine dentro del territorio nacional.

- Prohibir integralmente la publicidad, la promoción y el patrocinio en la mayor medida posible de acuerdo con sus constituciones nacionales.

Adecuando las disposiciones contempladas en el CMCT a la normativa constitucional vigente.

\section{Prevención del inicio del consumo}

La ONCT establecerá estrategias en los niveles de gobierno correspondientes para implementar las medidas pertinentes que permitan el cumplimiento de las metas relacionadas con el tema del inicio del consumo, reducción del consumo y espacios libres de humo.

En la actualidad el comportamiento de la epidemia del tabaquismo en la población mexicana revela los siguientes datos:

- Incremento en niños y adolescentes.

- Cada vez más productos dirigidos a las mujeres y mayor consumo por parte de éstas.

- Estabilidad en la prevalencia durante por lo menos los últimos 15 años.

- Mayor consumo en adolescentes.

- Alta intervención de la industria tabacalera para obstaculizar la implementación e instrumentación de políticas públicas.

Sabemos que la industria tabacalera ha centrado su atención en los jóvenes para "reemplazar" a los fumadores que fuman o mueren. Aun cuando cualquier persona 
que se expone al humo de tabaco puede desarrollar la adicción, las personas que no se han iniciado en el consumo antes de los 21 años, difícilmente comenzarán a fumar. Los adolescentes que experimentan con una sustancia tan altamente adictiva como es la nicotina están en riesgo de desarrollar la adicción de por vida. Los niños más pequeños que experimentan con el tabaco, constituyen la población más vulnerable y son los que muestran mayor probabilidad de convertirse en fumadores regulares y con menores probabilidades de abandonar el tabaco. ${ }^{4-46}$ Por lo anterior se hacen necesarias medidas tendientes a frenar este fenómeno. Algunas de las acciones girarán en torno a los siguientes lineamientos:

- Prevenir inicio en el consumo, especialmente en niños, adolescentes y mujeres.

- Ambientes $100 \%$ libres de humo:

o Se redactará la reglamentación necesaria

o Coordinación de las adecuaciones reglamentarias a que haya lugar

o Instrumentar e impulsar la correcta aplicación de la normatividad

o Difusión de la normatividad

o Se realizarán reuniones de cabildeo y abogacía con integrantes del Poder Legislativo en los tres niveles de gobierno para impulsar iniciativas que apoyen los espacios 100\% libres de humo de tabaco

o Coordinación de las acciones de información y capacitación para la instrumentación de los espacios libres de humo

o Alianzas con la industria de la hospitalidad (restaurantes, bares, discotecas, hoteles) para promocionar cumplimiento de la normatividad

o Desarrollo e implementación del sistema de vigilancia necesaria para el cumplimiento. Impulso de mecanismos sociales de vigilancia, denuncia y cumplimiento

o Elaboración de los documentos jurídicos necesarios para que la industria tabacalera cumpla con la normatividad relacionada

- Aumento de los impuestos.

- Prohibición de publicidad, promoción y patrocinio. Se busca evitar el interés de los adolescentes por los productos del tabaco y limitar de manera mediata el consumo.

- Reglamentación de los productos de tabaco.

\section{Reducción del consumo de productos del tabaco}

En el ámbito internacional es reconocido el impacto de diversas medidas de aplicación de políticas públicas y su impacto directo sobre la tasa de consumo de productos del tabaco. En este sentido, la experiencia señala las ventajas de una política fiscal adecuada, en especial destaca el aumento de los impuestos al tabaco como la medida más efectiva para reducir el consumo, ${ }^{21}$ lo que disminuye la accesibilidad de los cigarrillos especialmente entre los más jóvenes y grupos de bajos ingresos. Un incremento de $70 \%$ en el precio del tabaco, reduce una cuarta parte el número de muertes relacionadas con el tabaco. Por otra parte, el aumento de los impuestos al tabaco incrementa los ingresos fiscales. En general este tipo de medidas son bien aceptadas tanto por el público como por las autoridades, debido a que el tabaco no se considera un producto básico, de hecho se considera al tabaco como el producto más fácil de tasar impositivamente, aun entre la población de menores recursos, principalmente porque se le percibe como un producto nocivo para la salud..$^{9}$ En algunos países se utilizan los recursos provenientes del incremento de los impuestos al tabaco para la implementación del programa de control lo que ayuda a su aceptación inclusive entre los fumadores. ${ }^{47,48}$ Contrario a lo que la industria tabacalera argumenta contra este tipo de disposiciones, los ingresos fiscales de los gobiernos no disminuyen, al contrario, aumentan. De la misma manera, el aumento a los impuestos se asocia con una reducción en el consumo; un aumento de $10 \%$ en los impuestos disminuye el consumo en $4 \%$ en los países con más altos ingresos y aproximadamente $8 \%$ en los países con ingresos bajos o medios, mientras que la recaudación fiscal se incrementa en aproximadamente 7 por ciento. ${ }^{49}$

La implementación de leyes efectivas para el control del tabaco se acompaña de un incremento en la demanda de atención médica por una proporción de la población fumadora que se desiste de fumar como producto de las restricciones que enfrenta. Es así que uno de los aspectos centrales en materia de implementación de políticas públicas para el control del tabaco es lo relacionado con el acceso de los pacientes a los diferentes tratamientos para dejar de fumar. En el momento actual el CONADIC ha realizado diversos esfuerzos encaminados a la evaluación de la infraestructura y capacidad física instalada en el territorio nacional, se han realizado encuentros de clínicas de ayuda para dejar de fumar, así como reuniones de expertos en materia de tratamiento de la adicción a la nicotina. La responsabilidad de coordinar que los servicios de ayuda para dejar de fumar sean ofrecidos apropiadamente recae en el Sistema Nacional de Salud. La ONCT se encargará de monitorear los centros donde dichos tratamientos se encuentran disponibles, estandarizará la calidad de los servicios para garantizar que la atención sea óptima en cada uno de ellos. ${ }^{50}$ Se 
plantea realizar las siguientes acciones:

- Promover en el Sistema de Salud la Consejería (médicos, enfermeras, trabajadores sociales). ${ }^{51,52}$

- Incorporación del Primer Nivel de Atención como frente principal para la detección oportuna de pacientes fumadores e inicio de una intervención efectiva de manera temprana. ${ }^{53}$

- Incorporar el tema del tabaquismo como problema de salud pública prioritario para México en los planes y programas de estudio de todas las carreras contempladas dentro del campo de las ciencias de la salud. Fortalecer los contenidos que tratan el tema del tabaquismo en los libros de texto de educación básica, media y media superior, como una herramienta de prevención.

\section{Estrategias de comunicación y difusión}

La comunicación de los riesgos para la salud derivados del consumo de tabaco resulta de primordial importancia como estrategia de concienciación en los sectores de la sociedad civil. Como se ha mencionado previamente, a pesar de las evidencias disponibles en la actualidad, llama la atención que sean relativamente pocos los consumidores que tomen en cuenta los riesgos para su salud que implica el tabaquismo. Se recomienda hacer énfasis en la gran capacidad adictiva de la nicotina, se debe explicar de manera adecuada el espectro completo de los daños a la salud derivados del tabaquismo al público en general. La mayoría de las personas conocen los riesgos para la salud que implica el tabaquismo, sin embargo, se le sigue percibiendo como un "mal hábito". La incorporación de advertencias en las cajetillas constituye un vehículo apropiado para difundir información relevante que ha demostrado su utilidad. ${ }^{39}$

De la misma manera se hace necesaria la implementación de medidas de difusión para garantizar que la sociedad se encuentra informada respecto de los objetivos y los alcances de las medidas legislativas impulsadas por los tres niveles de gobierno. Estamos concientes de las acciones de la industria tabacalera para minar el impacto de las medidas encaminadas de control del tabaco. Dichas estrategias impactan sobre la opinión pública, creando confusión, controversia y falso debate, como el supuesto "derecho a fumar" o coartación de la "libertad de decisión". Por esta razón se considera prioritaria por parte de la ONCT la implementación de estrategias efectivas de comunicación y difusión a través de todos los medios disponibles.

En la actualidad se realizan esfuerzos desde diferentes instancias de los tres ámbitos de gobierno. El
Comité Interinstitucional de Lucha contra el Tabaco se ha encargado desde su creación de la organización de diferentes eventos alrededor de la celebración del Día Mundial sin Tabaco. De la misma manera, algunos de sus integrantes, dentro de los que destacan los Centros de Integración Juvenil y el Instituto Nacional de Enfermedades Respiratorias, han desarrollado estrategias efectivas de comunicación como son campañas en medios de comunicación, concursos de carteles alusivos al Día Mundial sin Fumar, diseño de material educativo específicamente dirigido a la comunicación de riesgos para la salud.

Se requiere generar un programa amplio y permanente para dar a conocer los diferentes métodos de ayuda para dejar de fumar, así como los sitios especializados donde se brindan dichos servicios.

La ONCT impulsará la continuidad de dichos esfuerzos y se encargará de replicar en los estados estrategias similares

\section{Sustentabilidad de la ONCT}

La ONCT se encargará de procurar la asignación suficiente y permanente de recursos para la sustentabilidad del Programa Nacional para el Control del Tabaco. Para lo anterior se gestionará ante las instancias correspondientes la asignación de recursos provenientes del presupuesto federal que garanticen la viabilidad del proyecto a largo plazo a través del Programa Operativo Anual (POA) del CONADIC y gestión ante instancias gubernamentales encargadas de la asignación del presupuesto federal. Gestionará reuniones periódicas con funcionarios de los tres ámbitos de gobierno para fortalecer la asignación de recursos al programa nacional de lucha contra el tabaco.

Diseminará la información relativa a los avances de la ONCT entre funcionarios de los tres ámbitos de gobierno para promover la sustentabilidad del proyecto.

Consolidar y mantener la cooperación internacional dando respuesta a los requerimientos y compromisos con organismos multilaterales y regionales.

\section{Discusión}

Por primera vez en la historia de nuestro país nos encontramos ante una coyuntura muy favorable para avanzar en el terreno del control del tabaco. El carácter de epidemia que ha adquirido el consumo de tabaco y las enfermedades asociadas obliga a implementar medidas estrictas de control que permitan disminuir la terrible carga que representa para el sistema de salud de nuestro país. Aunado al impacto en salud, es importante resaltar 
el impacto económico y social que implican las enfermedades producidas por el tabaquismo, el consumo crónico de tabaco incrementa los costos de la atención médica y resulta en pérdidas de productividad laboral, con efectos en la carga financiera de los fumadores, sus familiares, aseguradores y empleadores. ${ }^{19,20}$ Los costos indirectos también son sustanciales. Estimaciones de estos costos muestran que pueden llegar a ser hasta de 40 a $60 \%$ de los costos totales atribuibles al consumo de tabaco. Se ha estimado que en los países desarrollados las pérdidas de productividad corresponden aproximadamente a tres veces más los costos médicos directos. ${ }^{21,22}$

Otro elemento digno de tomarse en cuenta en el caso de México es la relativa estabilidad de la prevalencia de consumo de tabaco en población adulta, con un incremento en la población general que ha dado como resultado un aumento neto en el número total de fumadores. Este hecho es consistente con lo observado en otros países en desarrollo y su trascendencia radica en que si se mantienen las tendencias actuales, la epidemia del tabaquismo y las enfermedades asociadas se trasladará hacia dichos países, México incluido, incrementando la carga al sistema de salud, así como los costos directos e indirectos. ${ }^{1} \mathrm{~A}$ lo anterior habrá que sumarle el aumento en la prevalencia de consumo de cigarros entre los jóvenes mexicanos, especialmente entre las mujeres. ${ }^{54,55}$ Mientras que en 1988 la relación hombre: mujer era de 4:1, ésta disminuyó hacia 1998 para ubicarse en 2.6:1, la Encuesta Nacional de Adicciones versión 2002 reveló una relación hombre: mujer de 1.98:1, lo que sugiere un incremento sostenido en el número de fumadoras durante los últimos 15 años. ${ }^{10} \mathrm{En}$ estas circunstancias, nos enfrentamos a la necesidad de implementar medidas de control para evitar que en el mediano y largo plazo, la carga de la epidemia recaiga en las mujeres. En la mayoría de los países en desarrollo la prevalencia de consumo de tabaco entre las mujeres es aún baja en comparación con los hombres fumadores, sin embargo, tal como sucede con los jóvenes, la industria tabacalera dirige sus objetivos a las mujeres mediante campañas de publicidad agresivas por su potencial como futuras consumidoras. De hecho, el crecimiento actual de la epidemia del tabaquismo, se debe en parte al aumento en la prevalencia entre las mujeres de países con ingresos bajos y medios. Como lo señala la OMS en su más reciente informe sobre la epidemia global del tabaco, de mantenerse las tendencias actuales, en pocos años nos enfrentaremos a un problema de salud pública mucho peor, especialmente en los países en vías de desarrollo como México. ${ }^{9}$

México ha instrumentado, durante los últimos 15 años, acciones concretas para avanzar en materia de control de tabaco. Con la finalidad de optimizar los recursos y los esfuerzos, la Secretaría de Salud ha propuesto fortalecer la infraestructura nacional existente con la Oficina Nacional para el Control del Tabaco. La creación de esta oficina nos muestra la convicción para instrumentar y dar cumplimiento a los compromisos que plantea el Convenio Marco para el Control del Tabaco de la OMS. De igual forma la ONCT permitirá la consolidación de la capacidad nacional requerida para implementar un programa de control del tabaco a largo plazo. Se trata de un proceso interno para afrontar un reto mundial, el cual deberá implementarse con base al contexto del país, con una amplia participación del sector público y de la sociedad civil, en coordinación con los tres ámbitos de gobierno.

El problema del tabaquismo ha logrado una capacidad de cooperación y colaboración internacional sin precedentes. Se cuenta con herramientas de ayuda, así como con la experiencia obtenida en los países con mayor avance en el control del tabaco, de tal forma que es posible contar con instrumentos apropiados para la implementación de un programa de control acorde a las necesidades y a la realidad de cada país.

\section{Conclusiones}

Con la creación de la ONCT México avanza hacia la consolidación de un plan de acción en el campo de la salud pública. Sus tareas serán diversas, sin embargo, podemos resumirlas de la siguiente manera: analizará la situación nacional en aspectos de control de tabaco, voluntad política y percepción del problema de la población general; delineará las estrategias básicas para implementar un programa de control de tabaco acorde a la realidad del país; coordinará los esfuerzos nacionales en los tres ámbitos de gobierno, así como dentro del sector público y de las organizaciones de la sociedad civil; instrumentará programas de comunicación, educación y conciencia en la población general para generar el apoyo requerido de la sociedad; implementará la entrada en vigor de la Ley General para el Control del Tabaco; propiciará el apoyo de los tres ámbitos de gobierno para fortalecer los programas de control de tabaco y garantizar su desarrollo sustentable; ampliará la capacidad nacional para el control del tabaco, monitorizará las actividades de la industria tabacalera, en función de los obstáculos que pueda plantear para la aplicación de políticas públicas; finalmente, se encargará de evaluar el nivel de eficiencia de las políticas de control de tabaco.

Como se puede ver, se trata de una tarea ardua para estar a la vanguardia en materia de control de uno de 
los principales retos para la salud pública en los años por venir. Pero lo más importante, sin embargo, será conseguir los objetivos trazados que permitan disminuir en el mediano plazo la carga sobre el sistema de salud y sus repercusiones tanto económicas como sociales.

\section{Referencias}

I. Mathers CD, Loncar D. Projections of global mortality and burden of disease from 2002 to 2030. PLoS Medicine 2006;3(I I):e442.

2. Ezatti M, Lopez AD. Estimates of global mortality attributable to smoking in 2002. Lancet 2003;362(9387):847-852.

3. Consejo Nacional contra las Adicciones. Observatorio mexicano en tabaco, alcohol y otras drogas. México: Secretaría de Salud, 2003:63.

4. Peto $\mathrm{R}$, et al. Mortality from smoking worldwide. Br Med Bull 1996;52(I):I2-2I.

5. US Department of Health and Human Services. The health consequences of smoking: a report of the Surgeon General. Atlanta, US Department of Health and Human Services, Centers for Disease Control and Prevention, National Center for Chronic Disease Prevention and Health Promotion, Office on Smoking and Health [monografía en internet] 2004 [consultado $2 \mathrm{I}$ abril 2008]. Disponible en http://www.cdc. gov/tobacco/data_statistics/sgr/sgr_2004/chapters.htm

6. California Environmental Protection Agency. Proposed identification of environmental tobacco smoke as a toxic air contaminant: executive summary. Sacramento, California Environmental Protection Agency (2lpp) [monografía en internet] June 2005 [consultado 30 abril 2008]. Disponible en: ftp://ftp.arb.ca.gov/carbis/regact/ets2006/app3exe.pdf

7. US Department of Health and Human Services. The Health

Consequences of Involuntary Exposure to Tobacco Smoke: A Report of the Surgeon General-Executive Summary. US Department of Health and Human Services, Centers for Disease Control and Prevention, Coordinating Center for Health Promotion, National Center for Chronic Disease Prevention and Health Promotion, Office on Smoking and Health, 2006.

8. Guindon GE, Boisclair D. Past, current and future trends in tobacco use. Washington, DC: World Bank, 2003. Disponible en: http:// wwwl. worldbank.org/tobacco/pdf/Guindon-Past,\%20 current-\%20whole.pdf. 9. World Health Organization Report on the Global Tobacco Epidemic, 2008: The MPOWER package. Geneva: WHO, 2008.

10. Consejo Nacional contra las Adicciones. Encuesta Nacional de Adicciones 2002. Tabaco, alcohol y otras drogas. Resumen Ejecutivo. México: Secretaría de Salud, 2003

II. Peña-Corona GM, Kuri M, Tapia CR. El consumo de tabaco en México y Encuesta Nacional de Adicciones 1988 (Tabaco). México: Secretaría de Salud, 2000.

12. Gilmore A, et al. Prevalence of smoking in 8 countries of the former Soviet Union: results from the living conditions, lifestyles and health study. Am J Public Health 2004;94(I2):2177-2I87.

13. US Centers for Disease Control and Prevention. Global youth tobacco survey. Atlanta, US Centers for Disease Control and Prevention [monografía en internet], 2007 [consultado 30 abril 2008]. Disponible en http://www.cdc.gov/tobacco/global/gyts/datasets/policy.htm

14. Valdés-Salgado R, Reynales-Shigematsu LM, Lazcano-Ponce E, Hernández-Ávila E. Antes y después del Convenio Marco en México: una comparación desde la Encuesta sobre Tabaquismo en Jóvenes 2003 y 2006. Salud Publica Mex 2007;49(supl 2):155-169.

15. Guindon GE, et al. The cost attributable to tobacco use: a critical review of the literature. Geneva: World Health Organization, 2006. 16. US Centers for Disease Control and Prevention. Annual smoking- attributable mortality, years of potential life lost, and productivity losses - United States, 1997-200I. Morbidity and Mortality Weekly Report 2005;54(25):625-628.

17. Vázquez-Segovia LA, Sesma-Vázquez S, Hernández-Avila M. EI consumo de tabaco en los hogares en México: resultados de la Encuesta de Ingresos y Gastos de los Hogares, 1984-2000. Salud Publica Mex 2002;44(supl I):S76-S8I.

18. Sesma-Vázquez S, Campuzano-Rincón JC, Carreón-Rodríguez VG, Knaul F, López-Antuñano FJ, Hernández-Ávila M. El comportamiento de la demanda de tabaco en México: 1992-1998. Salud Publica Mex 2002;44(supl I):S82-S92.

19. Reynales-Shigematsu LM, Juárez-Márquez SA, Valdés-Salgado R. Costos de atención médica atribuibles al tabaquismo en el IMSS, Morelos. Salud Publica Mex 2005;47:45I-457.

20. Reynales-Shigematsu LM. Costos de atención médica de las enfermedades atribuibles al consumo de tabaco en las Américas: revisión de la literatura. Salud Publica Mex 2006;48(supl I):S190-S200.

2I. Max W. The financial impact of smoking on health-related cost: A review of the literature. Am J Health Promot 200I;I5(5):32I-33I. 22. Lightwood J, Collins D, Lapsley H, Novotny T. Estimating the costs of tobacco use. En: Jha P, Chaloupka F, ed. Tobacco control in developing countries. Oxford: Oxford University Press, 2000:63-99.

23. Organización Mundial de la Salud. Convenio Marco para el Control del Tabaco. Ginebra: Suiza [monografía en internet] 2003 [consultado 30 abril 2008]. Disponible en: http://www.who.int/gb/ebwha/pdf_files/WHA56/ sa56rl.pdf

24. Da Costa e Silva V, Nikogossian H. El Convenio Marco de la OMS: la globalización de la salud pública. Prevención del tabaquismo 2003;5(2): 7I-75.

25. World Health Organization. Building blocks for tobacco control: A handbook. Geneve, Switzerland [monografía en internet] 2004 [consultado 30 abril 2008]. Disponible en: http://www.who.int/tobacco/ resources/publications/tobaccocontrol_handbook/en/.

26. Wipfli H, Stillman F, Tamplin S, Da Costa e Silva VL, Yach D, Samet J. Achieving the Framework Convention on Tobacco Control's potential by investing in national capacity. Tob Control 2004;13(4):433-437.

27. Thompson F, Fagerström K. Current trends in international tobacco control. Clin Occup Environ Med. 2006;5(I):10I-I I6, ix.

28. Valdés Salgado R. El Convenio Marco para el Control del Tabaco. Salud Publica Mex 2003;45(3):153-154.

29. Valdés Salgado R. La ratificación del Convenio Marco para el Control del Tabaco. Salud Publica Mex 2004;46(3):202-203.

30. Economic and Social Council (ECOSOC). United Nations system to support capacity building. New York, 2002 (E/2002/58).

31. Fukuda-Parr S, Lopes C, Malik K, eds. Capacity for development: new solutions to old problems. London: Earthscan, 2002.

32. Tobacco Free Initiative/WHO Regional Office for Eastern Mediterranean (TFI-EMRO). Regional tobacco control profile. Geneva, World Health Organization [monografía en internet] [consultado 30 abril 2008]. Disponible en: http://www.emro.who.int/tfi/countryprofile.htm 33. Shafey O, Dolwick S, Guindon GE, eds. Tobacco Control Country Profiles 2003, American Cancer Society, Atlanta, GA, 2003.

34. Selin H, Pérez Martin J, Peruga A. Tobacco Control Country profiles: regional summary for the region of the Americas. World Health Organization, Tobacco Free Initiative, Regional Office for the Americas (PAHO). Tobacco Free Initiative 2003:32-33.

35. World Health Organization. Tobacco Free Initiative. Report of Activities 2003-2004. Geneve, Switzerland [monografía en internet] 2005 [consultado 30 abril 2008]. Disponible en http://www.who.int/tobacco/ resources/publications/tfi_final_26Jan.pdf

36. Guindon GE, Tobin S, Yach D. Trends and affordability of cigarette prices: ample room for tax increases and related health gains. Tobacco Control 2002; 11:35-43.

37. Estados Unidos Mexicanos. Diario Oficial de la Federación. Ley 
General de Salud. Título decimoprimero. 9 mayo 2007.

38. Cámara de Diputados. LX Legislatura. Comisión de Salud. Decreto que expide la Ley para el control del tabaco; $y$ deroga y reforma diversas disposiciones de la ley general de salud. México: 2006:22.

39. World Health Organization. Tobacco: deadly in any form or disguise. Geneva, World Health Organization [monografía en internet] 2006 [consultado 30 abril 2008]. Disponible en: http://www.who.int/tobacco/ communications/events/wntd/2006/Tfi_Rapport.pdf

40. Hammond D, et al. Effectiveness of cigarette warning labels in informing smokers about the risks of smoking: findings from the International Tobacco Control (ITC) Four Country Survey. Tobacco Control 2006;15(suppl 3):iii I 9-iii25.

4I. Saffer $H$, Chaloupka $F$. The effect of tobacco advertising bans on tobacco consumption. J Health Econ 2000; 19(6): I I 17- I I37.

42. Smee $\mathrm{C}$, et al. Effect of tobacco advertising on tobacco consumption: a discussion document reviewing the evidence. London: Economic and Operational Research Division, Department of Health, 1992.

43. Siahpush M, et al. Socioeconomic and country variations in knowledge of health risks of tobacco smoking and toxic constituents of smoke: results from the 2002 International Tobacco Control (ITC) Four Country Survey. Tobacco Control 2006;I5(suppl 3):iii65-iii70.

44.' Chen J, Millar WJ. Age of smoking initiation: implications for quitting. Health Reports 1998;9(4):39-46.

45. Khuder SA, Dayal HH, Mutgi AB. Age at smoking onset and its effect on smoking cessation. Addict Behav 1999;24(5):673-677.

46. Everett SA, et al. Initiation of cigarette smoking and subsequent smoking behavior among US high school students. Prev Med 1999;29(5):327-333.

47. Jha P, et al. Tobacco Addiction. In: Jamison DT, et al., eds. Disease control priorities in developing countries, $2^{\text {nd }}$ ed. New York, Oxford University Press and Washington, DC, World Bank [monografía en internet] 2006:869-885 [consultado 30 abril 2008]. Disponible en: http://files.dcp2.
org/pdf/DCP/DCP46.pdf

48. Armendares PE, Reynales Shigematsu LM. Política fiscal y control del tabaco: una oportunidad única para beneficiar a la salud pública y al erario. Salud Publica Mex 2006;48(supl I):SI67-SI72.

49. Jha P, Chaloupka FJ. Curbing the epidemic: governments and the economics of tobacco control [monografía en internet]. Washington, DC: World Bank 1999 [consultado 30 abril 2008]. Disponible en: http://www. globalink.org/tobacco/wb/wb04.shtml

50. Sansores R, Regalado J, Buitrago G. Guías Mexicanas para el Tratamiento del Tabaquismo. Un Consenso Nacional de Expertos. Neumol Cir Torax 2005;64(S2).

5I. Fiore MC. Treating tobacco use and dependence: a public health service clinical practice guideline [monografía en internet]. Rockville, MD, US Department of Health and Human Services, press briefing, 27 June 2000 [consultado 30 abril 2008]. Disponible en: http://www. surgeongeneral.gov/tobacco/mf062700.htm

52. Feenstra TL, et al. Cost-effectiveness of face-to-face smoking cessation interventions: a dynamic modeling study. Value in Health 2005;8(3): 178-190. 53. Tobacco Advisory Group of the Royal College of Physicians. Nicotine addiction in Britain; a report of the Tobacco Advisory Group of the Royal College of Physicians [monografía en internet]. London: Royal College of Physicians of London 2000 [consultado 30 abril 2008]. Disponible en: http://www.rcplondon.ac.uk/pubs/books/nicotine

54. Valdés-Salgado R, Meneses-González F, Lazcano-Ponce EC, HernándezRamos MI, Hernández-Ávila M. Encuesta sobre Tabaquismo en Jóvenes, México 2003. México: Instituto Nacional de Salud Pública, 2004 55. Valdés-Salgado R, Thrasher J, Sánchez-Zamorano LM, Lazcano-Ponde E, Reynales-Shigematsu LM, Menéses González F, et al. Los retos del Convenio Marco para el control del tabaco en México: un diagnóstico a partir de la encuesta sobre tabaquismo en jóvenes. Salud Publica Mex 2006;48(supl I):SS5-SI6. 